\title{
The Need for Artificial Intelligence in Digital Therapeutics
}

\author{
Adam Palanica $^{a} \quad$ Michael J. Docktor $^{b}$ Michael Lieberman ${ }^{a}$ Yan Fossat $^{a}$ \\ a Labs Department, Klick Health, Klick Inc., Toronto, ON, Canada; ${ }^{b}$ Division of \\ Gastroenterology, Boston Children's Hospital, Boston, MA, USA
}

\section{Keywords}

Digital therapeutics · Digital biomarkers - Digital medicine · Precision medicine · Artificial intelligence $\cdot$ Machine learning

\section{Abstract}

Digital therapeutics is a newly described concept in healthcare which is proposed to change patient behavior and treat medical conditions using a variety of digital technologies. However, the term is rarely defined with criteria that make it distinct from simply digitized versions of traditional therapeutics. Our objective is to describe a more valuable characteristic of digital therapeutics, which is distinct from traditional medicine or therapy: that is, the utilization of artificial intelligence and machine learning systems to monitor and predict individual patient symptom data in an adaptive clinical feedback loop via digital biomarkers to provide a precision medicine approach to healthcare. Artificial intelligence platforms can learn and predict effective interventions for individuals using a multitude of personal variables to provide a customized and more tailored therapy regimen. Digital therapeutics coupled with artificial intelligence and machine learning also allows more effective clinical observations and management at the population level for various health conditions and cohorts. This vital differentiation of digital therapeutics compared to other forms of therapeutics enables a more personalized form of healthcare that actively adapts to patients' individual clinical needs, goals, and lifestyles. Importantly, these characteristics are what needs to be emphasized to patients, physicians, and policy makers to advance the entire field of digital healthcare. 
Digital therapeutics have recently been described as a "new concept" in healthcare to change patient behavior and treat medical conditions using a variety of digital technologies [1-3]. The newly established Digital Therapeutics Alliance define digital therapeutics as delivering evidence-based, clinically validated therapeutic interventions for patients, driven by advanced technology and high-quality software programs to prevent, manage, or treat a medical disorder or disease [4]. They also describe how digital therapeutics must adhere to proper safety and efficacy protocols in randomized clinical trials, incorporate best practices for design and delivery, include patient privacy and security protections, publish clinically meaningful outcomes in peer-reviewed journals, and receive regulatory approval to support product claims of risk, efficacy, and intended use [4]. More recently, the Digital Medicine Society, Digital Therapeutics Alliance, HealthXL, and NODE. Health have also collaborated to clarify the definitions between digital health, digital medicine, and digital therapeutics to better support consumers, developers, and manufacturers of digital health tools [5].

However, upon teasing apart the definitions of digital therapeutics, it becomes apparent that the concept is not so new or different from traditional medicine and therapeutics. Drug manufacturers and healthcare providers have been incorporating these core principles into their everyday practices for decades. According to these criteria, "digital therapeutics" may very well be defined as "digital" versions of traditional "therapeutics;" the way that most researchers define the term is not more than the sum of its parts. By this definition, digital therapeutics simply involve software using generic fixed algorithms that do not change dynamically with each patient; they do not seem to take into account unique personalized patient symptom data to deliver adaptive interventions. For example, having a digital conversational agent (i.e., chatbot) provide cognitive behavioral therapy, medication adherence reminders, or online exercise and diet programs is conceptually similar to having a human healthcare provider provide those same therapies; only the delivery method (i.e., "messenger" of therapy) changes, but both digital programs and human therapists should provide the same predetermined treatment plan in patients. That is, both delivery methods provide the same generic therapy without adapting to individual patient genetics or lifestyles. Furthermore, in the case of the digital chatbot, a human clinician would still be needed for follow-up assessments to evaluate and modify the treatment regimen if needed, since the chatbot would not be able to adapt to individual patient differences.

We argue that the field of digital therapeutics should not only involve fixed or generic software, but rather focus on implementing more adaptive algorithms and flexible interventions via artificial intelligence and machine learning. Artificial intelligence and machine learning make it possible for systems to automatically learn from new experiences, adjust outputs, and perform human-like tasks without being explicitly programmed. In the current definitions of digital therapeutics [4, 5], there is no mention of artificial intelligence or selflearning, adaptable technologies. To be clear, we do not disagree with the current core principles of digital therapeutics [4,5], but we believe that digital therapeutics could be more novel, efficient, and beneficial to patients and providers if they implement more advanced forms of artificial intelligence in order to treat patients without the inherent need of a human supervisor.

Our aging population is growing rapidly, and this is associated with more and more chronic conditions. Consequently, physicians, nurses, and healthcare providers are experiencing growing demand with increased costs and long wait times. There are simply not enough medical professionals to take care of all the patients all the time, and these patients could benefit from ongoing personal support to manage their conditions and stay healthy. It only makes sense that digital therapeutics must apply more creative and technologically advanced tools to face these healthcare challenges. 
Therefore, an invaluable attribute of digital therapeutics should involve precision medicine - the customization of healthcare with medical diagnosis, treatments, and practices tailored to individual patients [6]. Precision medicine through digital therapeutics can be provided through the utilization of digital biomarkers and clinical feedback loop systems, which could offer continuous physiological monitoring and dynamic responses to control healthy homeostasis [7, 8]. Advanced artificial intelligence and machine learning systems can also incorporate hundreds of complex personal variables for each patient to provide more customized algorithms and interventions, which may be impossible for a human to evaluate.

The true essence of digital therapeutics, and the "new" value that it brings to healthcare, is not to merely supplement traditional treatments through companion software (e.g., mobile apps or biometric wearables), but rather provide a more personalized form of healthcare that actively adapts to individual patients' clinical needs, goals, and lifestyles. This differs from traditional therapeutic approaches, where a treatment regimen is designed for patients and then evaluated and modified only at periodic intervals (if ever), often months after the initial prescription. Traditional medications do not provide a closed-loop feedback mechanism that will adapt itself or change its dosage as the body reacts to surrounding environmental factors or the intervention itself. For example, an insulin injection may provide a quick solution to control blood glucose levels for patients with diabetes, but it cannot continuously react to the body as an adaptable "artificial pancreas" providing interactive glycemic control and insulin delivery [9-11]. With an artificial pancreas, a patient wears a continuous glucose monitor using an electrochemical or optical sensor for glucose measurements in the interstitial fluid (i.e., a digital biomarker); an algorithm is then established to correlate to actual blood glucose levels and carbohydrate intake, and based on these measurements, precise insulin dosing is automatically delivered by a wearable insulin pump [7]. This allows the patient to stay in a more tightly controlled glycemic window without the potential detriments of prolonged periods of hyper- or hypoglycemia.

Digital therapeutic technology allows for precision medicine at the patient-level in a way that a clinical team may not have the ability or time to accomplish for an individual patient, let alone an entire patient population. Put simply, the human body is not a static system, and precision medicine is needed to provide dynamic treatments to unique patients with continuously changing symptoms and reactions to medicine. Digital therapeutics should be able to deliver an automated, cost-effective, scalable, and convenient solution for patients 24 hours a day, including where resources and access may be scarce, such as remote and underserved locations. Digital therapeutics enable better clinical observations and measurements at scale to apply more impactful population health opportunities compared to traditional therapeutics. Healthcare providers often know very little about the time that patients spend outside of clinics; routine appointments with healthcare providers may be months or years apart between visits, and there is often recall bias from patients when trying to narrate their lives, who are usually only able to describe a rough history of the most recent few days or weeks. By contrast, digital therapeutic tools armed with artificial intelligence allows clinicians to better monitor as well as rapidly iterate and correct treatment options. While traditional medicine and therapeutics react to past behavior (i.e., diagnosing patients after they show symptoms), digital therapeutics can model future health states by incorporating artificial intelligence that is able to generate predictive interpretations of physiological data.

The improvements in machine learning, and specifically deep learning, over recent years have allowed new discoveries of many potential digital biomarkers of health and disease states [12]. These algorithms have demonstrated potential in identifying and predicting risk for patients with a variety of diseases and disorders, including diabetes, cardiovascular and gastrointestinal disease, neurological and movement disorders, mental health, and sleep disorders [12]. Consequently, research from the individual-patient level can be applied to 
Palanica et al.: Digital Therapeutics

larger populations for scalable understanding, management, and intervention of health conditions.

Given that the field of adaptable digital therapeutics is so new, very few examples of therapeutic technologies exist using advanced artificial intelligence that differ beyond diagnosing or monitoring tools (e.g., artificially intelligent radiology scanners). This gap in healthcare provides even more motivation for new technologies to be researched and developed for the automation of treating patients with precision medicine approaches. However, some potential therapeutic areas which could advance the machine learning capabilities include virtual reality systems to treat psychological disorders $[13,14]$, artificially intelligent inhalers to treat asthma or chronic obstructive pulmonary disease [15], or humanoid robots to help manage chronic diseases and medication adherence [16]. Advancing the computer intelligence of these systems would enable more efficient and practical use for patients on a regular basis.

Nevertheless, with all the potential benefits of advanced and "smart" forms of digital therapeutics, there are certainly limitations. Like traditional forms of medicine and therapy, the new field of digital therapeutics must adhere to regulatory guidelines and incorporate a compelling amount of scientific research to demonstrate accuracy, reliability, and validity of its concepts. The use of digital therapeutics by healthcare providers is only achievable after numerous substantiated, evidence-based claims, and a great deal of demonstrated safety to patients. Even the type of research itself may be a limitation to support the efficacy of digital therapeutic techniques; for example, using double-blind placebo-controlled trials (i.e., the gold standard of clinical studies) may not be entirely feasible, since these digital therapeutic tools are inherently different than traditional drugs. Furthermore, even if the scientific research is sound, the applications need to be implemented in the real world and be adopted with open arms by healthcare providers, patients, and consumers. Other research demonstrates that the implementation of wearable devices and data collection alone may not directly lead to behavior change; potential health outcomes related to digital therapeutic devices may strongly depend on motivational interventions, i.e., involving a "human touch" $[17,18]$. Usability and engagement factors would need to be vigorously tested to enhance the fluency and non-invasiveness of integration into patients' lives. Limitations to digital therapeutics also include privacy and accountability issues; depending on the context, it is difficult to say who will ultimately be responsible for patients' health, with both the artificial intelligence software and the prescribing physician of the digital therapeutic system having a vital role in patients' lives.

In conclusion, digital therapeutic systems have the potential to be a unique and efficient way to monitor symptoms, disease progression, and medication adherence, and provide relevant feedback interventions for patients and their clinical teams in various patient populations and disease states $[7,8,12]$. However, if digital therapeutics have a chance of being the "next best thing in healthcare," they need to offer something of significant value to patients and healthcare providers, besides simply encompassing digitized technology. Therefore, the need for artificial intelligence and digital biomarkers in digital therapeutics becomes a valuable differentiation from other forms of therapy that provides benefits above and beyond just simple digitized healthcare technology. The utilization of artificial intelligence systems allows for more convenient and effective monitoring and treatment of patient symptoms, and provides more time for physicians to spend with their patients for emotional, empathetic, and personal matters. Ultimately, when it comes to safety, efficacy, and level of evidence, digital therapeutics should be treated no differently than traditional therapeutics. Digital therapeutics should diverge regarding their clinical potential, both for symptom diagnosis and adaptive therapeutic benefits. Digital therapeutics plus artificial intelligence can adapt in real-time to the patient's current and predicted health state in a way that no traditional therapeutics, digitized or not, can achieve. 


\begin{tabular}{l|l}
\hline Digit Biomark 2020;4:21-25 \\
\hline DOI: 10.1159/000506861 & $\begin{array}{l}\text { (c) 2020 The Author(s). Published by S. Karger AG, Basel } \\
\text { www.karger.com/dib }\end{array}$ \\
\hline
\end{tabular}

Palanica et al.: Digital Therapeutics

\section{Disclosure Statement}

The authors declare that they have no conflicts of interest.

\section{Funding Sources}

This paper was internally funded and received no specific grant from any funding agency in the public, commercial, or not-for-profit sectors.

\section{Author Contributions}

A.P. wrote the first draft, with additional drafting by M.J.D, M.L., and Y.F. All of the authors contributed to the story development, as well as reviewing and editing the final content.

\section{References}

1 Joyce M, Leclerc 0, Westhues K, Xue H. Digital therapeutics: preparing for takeoff [cited 2020 Feb 10]. 2018. Available from: https://www.mckinsey.com/industries/pharmaceuticals-and-medical-products/ourinsights/digital-therapeutics-preparing-for-takeoff

2 Natanson E. Digital therapeutics: the future of health care will be app-based [cited 2020 Feb 10]. Forbes. 2017. Available from: https://www.forbes.com/sites/eladnatanson/2017/07/24/digital-therapeutics-the-futureof-health-care-will-be-app-based/\#5f9996117637

3 Sverdlov 0, van Dam J, Hannesdottir K, Thornton-Wells T. Digital therapeutics: an integral component of digital innovation in drug development. Clin Pharmacol Ther. 2018 Jul;104(1):72-80.

4 Digital Therapeutics Alliance. Digital therapeutics: combining technology and evidence-based medicine to transform personalized patient care [cited 2020 Feb 10]. 2018. Available from: https://www.dtxalliance.org/ wp-content/uploads/2018/09/DTA-Report_DTx-Industry-Foundations.pdf

5 Digital Medicine Society (DiMe). Digital Therapeutics Alliance (DTA), HealthXL, \& NODE. Health. Digital health, digital medicine, digital therapeutics (DTx): what's the difference? [cited 2020 Feb 10]. 2019. Available from: https://dtxalliance.org/2019/11/11/digital-health-digital-medicine-digital-therapeutics-dtx-whats-thedifference/

6 Ginsburg GS, Phillips KA. Precision medicine: from science to value. Health Aff (Millwood). 2018 May;37(5): 694-701.

7 Kovalchick C, Sirkar R, Regele OB, Kourtis LC, Schiller M, Wolpert H, et al. Can composite digital monitoring biomarkers come of age? A framework for utilization. J Clin Transl Sci. 2017 Dec;1(6):373-80.

8 Rodarte C. Pharmaceutical perspective: how digital biomarkers and contextual data will enable therapeutic environments. Digit Biomark. 2017 Aug;1(1):73-81.

9 Turksoy K, Bayrak ES, Quinn L, Littlejohn E, Cinar A. Multivariable adaptive closed-loop control of an artificial pancreas without meal and activity announcement. Diabetes Technol Ther. 2013 May;15(5):386-400.

10 Doyle FJ 3rd, Huyett LM, Lee JB, Zisser HC, Dassau E. Closed-loop artificial pancreas systems: engineering the algorithms. Diabetes Care. 2014;37(5):1191-7.

11 Bergenstal RM, Garg S, Weinzimer SA, Buckingham BA, Bode BW, Tamborlane WV, et al. Safety of a hybrid closed-loop insulin delivery system in patients with type 1 diabetes. JAMA. 2016 Oct;316(13):1407-8.

12 Dunn J, Runge R, Snyder M. Wearables and the medical revolution. Per Med. 2018 Sep;15(5):429-48.

13 Freeman D, Haselton P, Freeman J, Spanlang B, Kishore S, Albery E, et al. Automated psychological therapy using immersive virtual reality for treatment of fear of heights: a single-blind, parallel-group, randomised controlled trial. Lancet Psychiatry. 2018 Aug;5(8):625-32.

14 Freeman D, Lister R, Waite F, Yu LM, Slater M, Dunn G, et al. Automated psychological therapy using virtual reality (VR) for patients with persecutory delusions: study protocol for a single-blind parallel-group randomised controlled trial (THRIVE). Trials. 2019 Jan;20(1):87.

15 Rahman A. Top AI-powered asthma inhaler brands to lower the impacts of air pollution [cited 2020 Feb 10]. 2019. Available from: https://www.nsmedicaldevices.com/news/top-ai-asthma-inhaler-brands-air-pollution/

16 Park A. Machines treating patients? It's already happening [cited 2020 Feb 10]. 2019. Available from: https:// time.com/5556339/artificial-intelligence-robots-medicine/

17 Patel MS, Asch DA, Volpp KG. Wearable devices as facilitators, not drivers, of health behavior change. JAMA. 2015 Feb;313(5):459-60.

18 Noah B, Keller MS, Mosadeghi S, Stein L, Johl S, Delshad S, et al. Impact of remote patient monitoring on clinical outcomes: an updated meta-analysis of randomized controlled trials. NPJ Digit Med. 2018 Jan;1:20172. 